\title{
Cross-Feeding between Cyanobacterium Synechococcus and Escherichia Coli in Artificial Autotrophic-Heterotrophic Co-Culture System Revealed by Integrated Omics Analysis
}

\author{
Jiajia Ma \\ Tianjin University \\ Taohong Guo \\ Tianjin University \\ Lei Chen \\ Tianjin University \\ Xinyu Song \\ Tianjin University \\ Weiwen Zhang ( $\nabla$ wwzhang8@tju.edu.cn ) \\ Tianjin University
}

\section{Research Article}

Keywords: Artificial co-culture system, Interaction mechanism, Metabolomics, Quantitative proteomics, Transcriptomics, Cyanobacteria

Posted Date: December 13th, 2021

DOI: https://doi.org/10.21203/rs.3.rs-1147783/v1

License: (c) (i) This work is licensed under a Creative Commons Attribution 4.0 International License. Read Full License 


\section{Abstract}

Background: The light-driven consortia consisted of sucrose-secreting cyanobacteria and heterotrophic species capable of producing valuable chemicals have recently attracted significant attention, and are considered as a promising strategy for green biomanufacturing. In a previous study (Zhang et al, 2020, Biotechnol Biofuel, 13:82), we achieved a one-step conversion of $\mathrm{CO}_{2}$ through sucrose derived from cyanobacteria to fine chemicals by constructing an artificial co-culture system consisting of sucrosesecreting Synechococcus elongateus $\operatorname{cscB}^{+}$and 3-hydroxypropionic acid (3-HP) producing Escherichia coli ABKm. Analysis of the co-culture system showed that cyanobacterial cells were growing better than its corresponding axenic culture. To explore the underlaid mechanism and to identify the metabolic modules to further improve the co-culture system, an integrated metabolomics, transcriptomic and proteomic analysis was conducted.

Results: We first explored the effect of reactive oxygen species (ROS) on cyanobacterial cell growth under co-culture system by supplementing additional ascorbic acid to scavenge ROS in CoBG-11 medium. The result showed cyanobacterial growth was obviously improved with additional $1 \mathrm{mM}$ ascorbic acid under pure culture; however, cyanobacterial growth was still slower than that in the co-culture with E. coli, suggesting that the better growth of Synechococcus $\csc B^{+}$might be caused by other factors more than just ROS quenching. We then investigated the intracellular metabolite levels in cyanobacteria using LCMS based metabolomics analysis. The results showed that metabolites involved in central carbon metabolism were increased, suggesting more carbon sources were utilized by cyanobacteria in the coculture system, which illuminating that enhanced photosynthesis attributes to the higher $\mathrm{CO}_{2}$ availability produced from co-cultivated heterotrophic partner. To further explore the interaction based on crossfeeding and metabolite exchange, quantitative transcriptomics and proteomics were applied to Synechococcus $\csc B^{+}$. Analysis of differentially regulated genes/proteins showed that the higher availability of carbon, nitrogen, phosphate, calcium, $\mathrm{Cu}^{2+}, \mathrm{Fe}^{3+}$ and co-factors was observed in cocultivated Synechococcus $\csc B^{+}$during co-cultivation, suggesting the heterotrophic partner in the system might be involved in supplementing $\mathrm{CO}_{2}$ and improving essential micronutrients necessary to maintain high photosynthetic growth of Synechococcus $\csc B^{+}$.

Conclusion: Integrated omics analysis of the interaction mechanism between S. elongateus and E. coli showed metabolic changes such as enhanced photosynthesis, oxidative phosphorylation, essential micronutrients, and the ROS scavenging occurred at multiple levels of genes, proteins and metabolites, which might be together contributing to the better cell growth of Synechococcus $\csc B^{+}$in co-cultivation. In addition, the results implicated that the co-culture system could be further improved by engineering the modules related to the ROS quenching, carbon metabolism, nitrogen metabolism, Pi transport, metal transport and co-factors biosynthesis. Finally, the light condition, which may influence the cross-feeding metabolites between phototrophic and heterotrophic species, and also affect the oxidative pressure on the E. colistrains due to the photosynthesis, could be further optimized to improve cell growth in the coculture system, eventually leading to high productivity of value-added products. 


\section{Introduction}

Cyanobacteria with the capability of producing organic matter from $\mathrm{CO}_{2}$ by using solar energy, have attracted increased attention as environmentally friendly and sustainable "microbial cell factories" for the production of carbohydrate feedstocks to support traditional fermentation processes ${ }^{12}$. Take the sucrose, an easily fermentable feedstock, as example, several cyanobacterial species are capable of synthesizing and secreting sucrose as an osmolyte under appropriate environmental stimuli, such as osmotic pressure ${ }^{3}$, and this process can be sustained over a long period of time and at higher levels than that from plant-feedstock such as sugarcane and beet ${ }^{4,5}$. However, purification of sucrose from cyanobacterial cultivation supernatant is costly and the system is easily contaminated, which creates barriers to any scale-up cultivation ${ }^{6}$. In addition, any application of photosynthetic cell factories in scaleup facilities is always restricted by challenges from harsh environments, suggesting that the adaptability and compatibility of cyanobacterial cell factories should be further improved to facilitate the industrialscale biomanufacturing ${ }^{7}$. In recent years, increasing evidences suggested that the exchange of essential metabolites between microorganisms could be a crucial process that can significantly affect growth, composition and the structure stability of microbial communities in nature ${ }^{8,9}$. In aquatic environments, the ecological interaction between photo-autotropic and heterotrophic species is based on cross-feeding and metabolite exchange ${ }^{10}$. In this case, the photo-autotrophs excreted material ranging from targeted photosynthetic intermediates such as glycolate, osmolytes and fatty acids, and extracellular polymeric substance, to the products of cell lysis that can include sugars, proteins, lipids and nucleic acids ${ }^{11,12}$. In exchange, heterotrophic species are thought to provide essential micronutrients, such as vitamins, amino acids and bioavailable trace metals, necessary to maintain high photosynthetic productivity ${ }^{9}$. In addition, the positive effects on the autotrophs were also observed, which might be attributed to the decrease of oxidative stress by heterotrophs through reactive oxygen species (ROS) scavenging ${ }^{13,14}$. Inspired by the symbiotic system commonly found in nature, increasing efforts have been made in recent years to design artificial routes of metabolite interchange in order to construct new symbiotic systems with high efficiency and stability 15,16 .

The light-driven artificial consortia consisted of sucrose-secreting cyanobacterium and heterotrophic species have recently attracted significant attention as the alternatives for the utilization of sucrose from cyanobacteria ${ }^{17}$. For example, Ducat et al. constructed a co-culture system consisting of the cyanobacterium Synechococcus elongatus PCC 7942 (hereafter as Synechococcus 7942) and the heterotrophic bacterium Halomonas boliviensis, in which the growth of $H$. boliviensis was supported by sucrose produced by $S$. elongatus $7942^{18}$. In another study, Li et al. designed a co-culture system with the sucrose-secreting S. elongatus 7942 and three different yeasts to mimic lichen and research the interaction between the autotrophic and heterotrophic strains ${ }^{13}$. More recently, Liu et al. constructed a coculture system composed of $S$. elongatus 7942 and $E$. coli to produce isoprene and extended the fermentation time of co-cultivation was extended from $100 \mathrm{~h}$ to $400 \mathrm{~h}$ by adjusting the inoculation ratio between S. elongatus 7942 and E. coli, in which the production of isoprene was increased sevenfold to 
$0.4 \mathrm{~g} / \mathrm{L}$ compared to the axenic culture of $E$. coli $^{19}$. In addition, cyanobacteria other than model species were also utilized, for example, Zhang et al. constructed a microbial consortium consisting of the fastgrowing cyanobacterium Synechococcus elongatus UTEX 2973 recently identified (hereafter as Synechococcus 2973) which are capable of growing under high light and temperature ${ }^{20}$, as well as $E$. coli to sequentially produce sucrose and then the platform chemical 3-hydroxypropionic acid (3-HP) from $\mathrm{CO}_{2}{ }^{21}$. All these studies enlightened us that light-driven co-culture system could be a promising strategy for future $\mathrm{CO}_{2}$ based biomanufacturing.

To construct light-driven co-culture systems with high efficiency, it is necessary to fully understand the metabolic mechanism underlaid the interaction between autotrophs and heterotrophs. Although several previously studies have showed that the cyanobacterial cell growth could be improved in co-culture system ${ }^{13}$, the mechanism is yet to be determined. Moreover, while it is fully expected that the mechanism involves more than just single gene, or even single metabolic module, so far only a few studies utilized global-based omics techniques to explore the interaction mechanism at multi-level of RNA, protein and metabolite ${ }^{22-24}$. Due to the complexity of co-culture structure, the challenge to study on the interaction mechanism is also increased, integrated omics analysis could be a good approach to obtain a

"panorama" of cells in the co-culture systems and reveal novel insights into the biological mechanism ${ }^{25}$. For example, Amin et al. analyzed the signaling and interaction between diatom and associated bacteria through integrated metabolite and transcriptomic analysis, in which the tryptophan and indole-3-acetic acid were determined as the key signaling molecules involving in the complex exchange of nutrients ${ }^{26}$, demonstrating that the approach of integrated transcriptome, proteome and metabolome should be adopted to explore microbial interactions in the co-culture systems.

In our previous study, Zhang et al. ${ }^{21}$ constructed an artificial co-culture system composed of the sucrosesecreting strain Synechococcus elongatuscscB $B^{+}$and the sucrose-utilizing and 3-hydroxypropionic acid (3HP) -producing strain E. coli ABKm. The system was able to produce $\sim 68.29 \mathrm{mg} / \mathrm{L} 3-\mathrm{HP}$ in 7 days. In spite of the one-step sucrose utilization co-culture system was successfully constructed, productivity and stability of the co-culture systems remain challenging. In this study, an integrated proteomics and transcriptomics approach was employed to analyze the metabolic responses of cyanobacteria to the heterotrophic partner in the artificial co-culture system, which will be valuable to identify the metabolic modules involved in efficiency and stability of the co-culture system and apply them as potential engineering targets to further optimize the system, as well as for guiding cultivation optimization.

\section{Methods}

\subsection{Strains and culture conditions}

The sucrose-secreting strain SynechococcuscscB ${ }^{+}$(derived from Synechococcus elongatus UTEX 2973) and the sucrose-utilizing and 3-HP-producing E. coli ABKm strain reported in our previous study were used to construct co-culture system ${ }^{21}$. Synechococcuscsc $B^{+}$was cultivated under $100 \mu$ mol photons $\mathrm{m}^{-2} \mathrm{~s}^{-1}$ in 
an illuminating shaking incubator (HNYC-202T, Honour, Tianjin, China) at $130 \mathrm{rpm}$ and $37^{\circ} \mathrm{C}$ or on BG-11 agar plates in an incubator (SPX-250B-G, Boxun, Shanghai, China) ${ }^{27}$. E. coli ABKm strain were grown on LB medium or agar plates with appropriate antibiotics added to maintain plasmids at $37^{\circ} \mathrm{C}$ in a shaking incubator (HNY-100B, Honour, Tianjin, China) at $200 \mathrm{rpm}$ or in an incubator, respectively. Co-culture medium (hereafter as CoBG-11) was used to construct co-culture system according to the previous study 21, in which $150 \mathrm{mM} \mathrm{NaCl}, 4 \mathrm{mM} \mathrm{NH}_{4} \mathrm{Cl}$ and $3 \mathrm{~g} / \mathrm{L} \mathrm{2-[[1,3-dihydroxy-2-(hydroxymethyl)} \mathrm{propan-2-yl]} \mathrm{amino]}$ ethanesulfonic acid (TES) were supplemented into the BG-11 medium. The pH value was adjusted with $\mathrm{NaOH}$ to 8.3 .

For construction of co-culture system, the exponential phase SynechococcuscscB $B^{+}\left(\mathrm{OD}_{750} \approx 1.0\right)$ was collected and inoculated into $25 \mathrm{~mL}$ of CoBG-11 and grown at $30{ }^{\circ} \mathrm{C}$ for $48 \mathrm{~h}$ to an $\mathrm{OD}_{750}$ of 0.5 . E. coli was cultivated in CoBG-11 with $1 \mathrm{~g} / \mathrm{L}$ sucrose for $48 \mathrm{~h}$, and then the cells were collected and resuspended in deionized water and inoculated into the $25 \mathrm{~mL}$ SynechococcuscscB ${ }^{+}$culture grown on CoBG-11. To separate two species in the co-culture system, the dialysis bag (diameter is $36 \mathrm{~mm}$, molecular weight cutoff is $14 \mathrm{kDa}$, respectively, biosharp, Hefei, China) was used. The E. coli ABKm was incubated in the dialysis bag, while the SynechococcuscscB ${ }^{+}$was incubated outside in the flask. The pre-treatment of dialysis bags was according to a previous study with some modifications ${ }^{28}$. Briefly, the dialysis bag was cut into small pieces of appropriate length (approximately $10 \mathrm{~cm}$ ), which were boiled for 10 minutes with a large volume of $1 \mathrm{mmol} / \mathrm{L} \mathrm{EDTA}(\mathrm{pH} 8.0)$. And then the dialysis bags were boiled with distilled water for 10 minutes for twice. The prepared dialysis bag was autoclave sterilized before using.

The cell density was measured at $\mathrm{OD}_{750}$ using a UV-1750 spectrophotometer (Shimadzu, Kyoto, Japan). The co-cultivated SynechococcuscscB $B^{+}$was counted by a hemocytometer under a microscope (BX43, Olympus, Shinjuku, Tokyo, Japan) after series dilution.

\subsection{Determination of $\mathrm{H}_{2} \mathrm{O}_{2}$ concentration}

$\mathrm{H}_{2} \mathrm{O}_{2}$ Quantitative Assay Kit (Water-Compatible) (Sangon Biotech, Shanghai, China) was used to analyze the content of $\mathrm{H}_{2} \mathrm{O}_{2}$ in the medium supernatant. Under acidic conditions, $\mathrm{H}_{2} \mathrm{O}_{2}$ oxidizes $\mathrm{Fe}^{2+}$ ions into $\mathrm{Fe}^{3+}$ ions, which then combine with dye molecules to form $\mathrm{Fe}^{3+}$-dye complex. The formed complex has the maximum absorption wavelength at $560 \mathrm{~nm}$ (or $595 \mathrm{~nm}$ ) and the absorption value is proportional to the concentration of $\mathrm{H}_{2} \mathrm{O}_{2}$, which was detected by spectrophotometer (Thermo Fisher Scientific Oy, Vantaa, Finland).

\subsection{LC-MS based metabolomics analysis}

Liquid chromatography-mass spectrometry (LC-MS) based targeted metabolomics was performed according to the protocol described previously ${ }^{29}$. Cells $\left(5 \mathrm{OD}_{730}\right.$ unit) were harvested at $48 \mathrm{~h}$ via centrifugation at $7380 \mathrm{rpm}$ for $5 \mathrm{~min}$ at $4{ }^{\circ} \mathrm{C}$ (Eppendorf 5430R), quenched, and extracted rapidly with 900 $\mu \mathrm{L}$ of $80: 20$ methanol/water $\left(\mathrm{v} / \mathrm{v} ;-80^{\circ} \mathrm{C}\right.$ pretreated) and then frozen in liquid nitrogen. Intracellular 
metabolites were extracted via the freeze/thaw cycle for three times. The aforementioned extraction process was repeated with another $500 \mu \mathrm{L}$ 80:20 methanol/water $(\mathrm{v} / \mathrm{v})$. The supernatant was combined and filtered through a $0.22 \mu \mathrm{m}$ syringe filter. The solvents were removed using a vacuum concentrator system (ZLS-1, Hunan, China), and $100 \mu \mathrm{L}$ of ddH2O was added and mixed well. LC-MS analysis was conducted using an Agilent 1260 series binary HPLC system equipped with a Synergi Hydro-RP (C18) 150 $\mathrm{mm} \times 2.0 \mathrm{~mm}$ ID, $4 \mu \mathrm{m} 80 \AA \AA$ particle column (Phenomenex, Torrance, CA, U.S.A.), and an Agilent 6410 triple quadrupole mass analyzer equipped with an electrospray ionization (ESI) source. Data were acquired using the Agilent Mass Hunter work-station LC/QQQ acquisition software (version B.04.01), and chromatographic peaks were subsequently integrated via the Agilent Qualitative Analysis software (version B.04.00). All data of metabolomic profiling was first normalized by the internal control and the cell numbers of the samples. Each condition analysis consisted of four biological replicates and three technical replicates.

\subsection{Transcriptomic analysis of cyanobacterial responses to $E$. coli in co-culture system}

Considering the characteristics of transcriptomics technology and the accuracy of transcriptomics data, dialysis bags were used to separate cyanobacteria and $E$. coli to construct the co-culture system. For transcriptomic analysis, $5 \mathrm{OD}_{750}$ of co-cultured and ascorbic acid treated axenic SynechococcuscscB ${ }^{+}$ were collected respectively for extracting RNA samples; meanwhile, the same amount of SynechococcuscscB ${ }^{+}$cultivated under axenic was used as control. The transcriptomics analysis was conducted by GENEWIZ (Suzhou, China). There three biological replicates for each sample, and two statistic parameters which are fold change $>1.5$ and $Q$-value (fdr or padj) $\leq 0.05$ were used to determine differentially regulated genes.

\subsection{Quantitative proteomics analysis of cyanobacterial responses to $E$. coli in co-culture system}

The same weight of four-day co-cultured strains were sampled for proteome analysis. The samples were enzymatically digested by trypsin, following marked by isobaric tags for relative and absolute quantification (iTRAQ) technique, the samples were analyzed by liquid chromatography-tandem mass spectrometry (LC-MS/MS). The axenic Synechococcus 2973 cells with same incubation time were used as control. The technical service and data of quantitative proteomics were provided by BGI (Shenzhen, China). Three biological replicates for each sample were used. In the case of unmatched biological replicates, two statistic parameters, fold change $>1.2$ (the average ratio of the nine comparison groups) and $P$-value $<0.05$ (t-test of nine comparison groups) were used to screen differentially regulated proteins.

\subsection{Quantitative real-time PCR analysis}

For RNA extraction, the $2 \mathrm{OD}_{750}$ cells of Synechococcus $\csc B^{+}$under axenic culture and co-culture were collected and centrifuged at 7,830 rpm and $4{ }^{\circ} \mathrm{C}$ for 5 mins. The total RNA samples were extracted using Direct-zol ${ }^{\text {TM }}$ RNA Miniprep kit (ZYMO RESEARCH, CA, USA) according to the instruction, and then reverse transcribed as cDNA template using HiScript ${ }^{\circledR}$ II Q RT SuperMix for qPCR (+gDNA wiper) reagent 
(Vazyme, China). The quantitative real-time PCR (qPCR) reactions were performed according to the methods described previously ${ }^{30}$. Briefly, the $10 \mu \mathrm{L}$ reaction system was composed of $5 \mu \mathrm{L}$ of $2 \times$ ChamQ Universal SYBR qPCR Master Mix (Vazyme, China), $3 \mu \mathrm{L}$ of RNase-free $\mathrm{H}_{2} \mathrm{O}, 0.5 \mu \mathrm{L}(5 \mu \mathrm{M})$ of each upstream primer and downstream primer and $1 \mu \mathrm{L}$ of appropriately diluted cDNA template. The relative changes in gene expression from qPCR experiment could be analyzed using the $2^{-\triangle \triangle C T}$ method ${ }^{31}$.

\section{Results And Discussion}

\subsection{Effect of ROS quenching on cyanobacterial cell growth in co-culture system}

With the potential to react with biomolecules including nucleic acids, proteins and lipids, ROS can cause oxidative stress to cells, leading to cellular damage ${ }^{32}$. Previous studies pointed to the possibility that heterotrophic bacteria can remove ROS in medium to improve cell growth of cyanobacteria in co-culture system, eventually enhancing the stability of co-culture system ${ }^{13,14}$. To verify this hypothesis, ascorbic acid was added into CoBG-11 medium to decrease ROS generation through directly scavenge $\mathrm{O}_{2}{ }^{\circ},{ }^{\circ} \mathrm{OH}$ and reduce $\mathrm{H}_{2} \mathrm{O}_{2}$ to water ${ }^{33}$. Different concentrations of ascorbic acid, $0.1 \mathrm{mM}, 1 \mathrm{mM}, 2 \mathrm{mM}$ were added into pure cultural SynechococcuscscB ${ }^{+}$respectively in CoBG-11 34, 35, the analysis showed that SynechococcuscscB ${ }^{+}$grew better with additional $1 \mathrm{mM}$ ascorbic acid in CoBG-11 compared with $0.1 \mathrm{mM}$ ascorbic acid supplementation, while cell growth was inhibited after three days when supplemented 2 mM ascorbic acid (Fig. 1A). Consistently, the analysis showed that $\mathrm{H}_{2} \mathrm{O}_{2}$ content was significantly decreased with ascorbic acid added (Fig. 1B); however, it is unclear why the cells growth was arrested when $2 \mathrm{mM}$ supplementary ascorbic acid led to almost no detectable $\mathrm{H}_{2} \mathrm{O}_{2}$ at 4 days. More importantly, although cyanobacterial growth was obviously improved with additional $1 \mathrm{mM}$ ascorbic acid under pure culture, cyanobacterial growth was still slower than that in co-culture with $E$. coli, suggesting that the better growth of SynechococcuscscB ${ }^{+}$might be caused by other factors more than just ROS quenching.

\subsection{Analysis of key metabolites in S. elongateus $\operatorname{cscB}^{+}$during co-cultivation by target LC-MS metabolomics}

As the stability and productivity in the co-culture system was dependent on the cyanobacterial sucrose production, intracellular levels of key metabolites within Synechococcus $\csc B^{+}$cells were investigated. LC-MS based metabolomics approach, which has been employed previously to comparatively analyze cellular metabolism in the engineered cyanobacterial strains ${ }^{29,36}$, was applied to compare co-cultivated and pure cultural Synechococcus $\csc B^{+}$. As shown in Fig. 2, twenty-one metabolites of cyanobacterial metabolism involve in glycolysis, amino acid, and the citric acid (TCA) cycle were chemically classified. Comparative analysis showed that the intracellular contents of FBP, F6P, E4P, R5P and acetyl-CoA were increased, suggesting more carbon sources were utilized in co-cultivated Synechococcus $\csc B^{+}$. Five amino acids, lysine (Lys), serine (Ser), valine (Val), alanine (Ala) and phenylalanine (Phe) were found with significant up-regulation during co-cultivation condition. Three metabolites involved in TCA cycle including citric, malate and succinate, were also showed significant increases in co-cultivation condition. 
One possible explanation for the increased metabolites in glycolysis, amino acid, and TCA cycle is that increasingly available $\mathrm{CO}_{2}$, possibly from the respiration of heterotrophic cells, contributes to cyanobacterial better cell growth during co-cultivation, consistent with a previous finding that enhanced $\mathrm{CO}_{2}$ fixation and oil production in co-culturing green algal Chlorella and yeast Saccharomyces cerevisiae system ${ }^{37}$. The results were also consistent with a more recent study which found salt stress redirect the fixed $\mathrm{CO}_{2}$ toward sucrose production rather than biomass and glycogen accumulation in engineered Synechococcus $2973^{38}$. In addition, the results of LC-MS also implicated that the enhanced $\mathrm{CO}_{2}$ fixation could be used as an engineering target for further improving sucrose production in the co-cultivated cyanobacteria by modulating sucrose production pathway.

\subsection{Analysis of cyanobacterial metabolic responses to $E$. colin co-cultivated $S$. elongateusby transcriptomics}

For the interaction based on cross-feeding and metabolite exchange, early studies have shown that heterotrophic bacteria could also be involved in providing essential micronutrients, such vitamins, amino acids and bioavailable trace metals, necessary to maintain high photosynthetic productivity ${ }^{39}$. To explore the mechanism underlaid the increased cell growth of co-cultivated cyanobacteria, and to determine the factors necessary for the stability and fermentation performance, transcriptomics between pure cultural cyanobacteria (C) and co-cultural cyanobacteria with E. coli (D) was applied to analyze the interaction mechanism of cyanobacteria responses to the heterotrophic partner in co-culture system. With a cutoff of 1.5 -fold change and a $p$ value of statistical significance less than 0.05 , we found 120 genes up-regulated and 104 genes down-regulated as a result of co-cultivation, respectively. The reliability and accuracy of the transcriptomics data was independently verified by real-time quantitative PCR (qRT-PCR) (Suppl Table S1), the correlation coefficient $R^{2}$ was 0.9086 (Fig. S1), indicating the transcriptomics data collected in this study is of very high accuracy. The analysis showed that a large fraction of up-regulated transcripts was affiliated with photosynthesis and oxidative phosphorylation (25\%), signal transduction and membrane transport (13\%), translation (10\%), genetic information processing (6\%), metabolism of cofactors and vitamins (3\%) role categories (Fig. 3A, Suppl Table S2), suggesting the key central metabolism of cyanobacterial cells was affected by the presence of $E$. coli in the co-culture system.

The analysis showed significant increases in transcripts levels of phosphate transport system permease genes M744_04015 (pstS, sphX), and M744_04030 (pstA), and iron transporter (for example, bicarbonate transporter M744_01215, calcium/sodium antiporter M744_01815, P-type $\mathrm{Cu}^{2+}$ transporter M744_04705), suggesting higher availability of these important micronutrients for Synechococcus cscB ${ }^{+}$during cocultivation condition ${ }^{40}$. Meanwhile, the gene M744_08450 ( $\mathrm{cmpD}$ encoding a bicarbonate transporter) was significantly down-regulated, which illuminated that $\mathrm{CO}_{2}$ availability might be increased due to the fact that $E$. coli $\mathrm{ABKm}$ might provide additional $\mathrm{CO}_{2}$ for co-cultivated Synechococcus $\mathrm{cscB}^{+}$. In addition, M744_08660 $(\bmod A)$ encoding a molybdate transport system substrate-binding protein was also found down-regulated. Molybdenum is an essential component of the cofactors of many metalloenzymes including nitrate reductase and Mo-nitrogenase ${ }^{41}$, the down-regulation of $\bmod A$ might suggest that more 
nitrogen available for Synechococcus $\csc ^{+}$during co-cultivation as well. Varkey et al. described the upregulation in several translational, ribosomal biogenesis and transcriptional proteins under oxidative stress in marine Synechococcus isolates ${ }^{42}$. However, many transcripts involved in ribosomal proteins (M744_13675, M744_13670, M744_05205, M744_00735, M744_05210, M744_12320, M744_05195, M744_05180, M744_05185), tRNA synthetases (M744_03935, M744_5340) and RNA binding protein (M744_12800) were down-regulated in our study, which likely due to the reduced ROS content in coculture system ${ }^{13}$. The down-regulated transcripts in co-cultivated Synechococcus $\csc B^{+}$compared with under axenic condition were also demonstrated (Suppl Table S3).

Consistent with abovementioned results, significant decreases in transcripts levels of oxidative stress related genes were found in co-cultivated Synechococcus cscB ${ }^{+}$, including two genes (M744_11065 and M744_01810) encoding high light-inducible proteins (HIi), M744_03995 encoding antioxidant protein and M744_07160 encoding heme oxygenase, indicating possible reduced oxidative stress in co-cultivated Synechococcus $\csc B^{+}$. High light-induced proteins are critical for the energy dissipation mechanism to resist oxidative stress in cyanobacteria ${ }^{43}$. He et al. found the gene expression of 4 hlis genes were induced under low temperature, strong light stress and nutrient deficiency condition in Synechocystis sp. PCC 6803 (hereafter as Synechocystis 6803), and the hli knockout mutant strain could not be survival under strong light, suggesting that the high light-induced protein may play a photoprotective role ${ }^{44}$. The ferredoxin-dependent heme oxygenase catalyzes the degradation of heme to produce biliverdin IXa with the release of ferrous iron ${ }^{45}$. Cheng et al. found the down-regulation of heme oxygenase gene might reduce the release of detrimental free iron that causes oxidative stress ${ }^{46}$. Consistent with these results, our finding that the down-regulation of these four genes that the better growth of Synechococcus cscB $^{+}$in co-culture system was partially attributed to the quenching of ROS by heterotrophic partner $E$. coli ABKm. In a previous study, Vance et al. found that the phospholipid/cholesterol/gamma-HCH transport system permease protein (MlaE) was down-regulated after exposure to a high bisphenol A concentration, which might inhibit phospholipid transport, and subsequently altered the spontaneous diffusion of the membrane to eventually caused membrane damage ${ }^{47}$. Interestingly, the relative expression of M744_01095 ( $\mathrm{m} / \mathrm{aE}$ ) was also increased in co-cultivated Synechococcus $\mathrm{cscB}^{+}$, which also suggested that ROS induced membrane damage was relieved by the presence of the heterotrophic partner.

In cyanobacteria, the secretory (Sec) pathway is critical for proteins transportation across the plasma membrane and thylakoid membrane ${ }^{48}$. The core of translocase in Sec pathway is a protein channel assembled by heterotrimeric membrane protein complex SecYEG and ATPase SecA oligomers, SecA is used as a molecular motor ${ }^{49}$. It was estimated that $82 \%$ of translocated proteins in Synechocystis 6803 contain a Sec signal peptide ${ }^{50}$. The expression of M744_13645 (secE) was up-regulated in co-cultivated Synechococcus CscB ${ }^{+}$. In addition, the relative expression of M744_09155 (yidC) was increased in cocultivated Synechococcus $\operatorname{cscB}^{+}$as YidC protein mediates integration of membrane integral proteins in bacteria and thylakoid membrane ${ }^{51}$. The increased expression levels of $s e c E$ and $y i d C$ were consisted 
with the phenotype of better cyanobacterial growth as translocase is responsible for the insertion of the photosystem integral membrane proteins into the thylakoid membrane in cyanobacteria ${ }^{52}$.

\subsection{Analysis of cyanobacterial metabolic responses to $E$. colin co-cultivated $S$. elongateusby quantitative proteomics}

The low correlation between mRNA and protein expression has been found and well discussed in previous studies, which might be caused by the widespread post-transcriptional regulation mechanism ${ }^{53}$, 54 . For example, Nie et al. found that correlation of mRNA expression and protein abundance was affected at a fairly significant level by multiple factors related to translational efficiency ${ }^{55}$. In order to fully identify the interaction mechanism in the co-culture system, the quantitative iTRAQ proteomics was used to analyze cell responses of Synechococcus $\csc B^{+}$adapt to $E$. coli in co-culture system. Three Synechococcus $\csc B^{+}$samples from the co-culture (E1, E2, E3) and three from the axenic culture (C1, C2, C3) were collected after cultivation of $96 \mathrm{~h}$, respectively, and the differential profiles of proteins in Synechococcus $\operatorname{cscB}^{+}$were identified by setting comparison groups of E1 vs. C1, E1 vs. C2, E1 vs. C3, E2 vs. C1, E2 vs. C2, E2 vs. C3, E3 vs. C1, E3 vs. C2, E3 vs. C3. The proteomic analysis identified a total of 914,635 spectra, among which 21,603 unique spectra met the 1\% FDR filter and were matched to 2,206 unique proteins in the genome. In addition, a good coverage was obtained for a wide MW range for protein (Fig. 4A). Most of the identified proteins were with good peptide coverage, $\sim 89 \%$ of the proteins were with more than $10 \%$ of the sequence coverage and $~ 87 \%$ were with more than $20 \%$ of the sequence coverage (Fig. 4B). Among the functional categories, the "general function prediction only" was the top detected functional category, representing $13.43 \%$ of all the identified protein (Fig. $4 \mathrm{C}$ ). This result is consisted with the previous finding that approximately $45 \%$ of proteins in the cyanobacterial genome are hypothetical proteins ${ }^{56}$. Other frequently detected functional categories included "translation, ribosome structure and biogenesis" (9.42\%), "amino acid transport and metabolism" (8.67\%), "posttranslational modification, protein turnover, chaperones" (8.51\%), "signal transduction mechanism" (7.17\%), "carbohydrate transport and metabolism" (6.51\%).

All 251 differentially expressed proteins were divided into 21 secondary branches of pathways based on the KEGG database classification, in which 181 differentially regulated proteins are related to cell metabolism, including energy metabolism (10.36\%), metabolism of cofactors and vitamins $(10.36 \%)$, carbohydrate metabolism (10.36\%), and amino acid metabolism (4.38\%) (Fig. 5A). The number of upand down-regulated proteins in each annotated pathway was shown in Fig. 5B. The KEGG enrichment analysis suggested that seven pathways were significantly enriched $(P$-value $<0.05)$ in the up-regulated differential proteins, including "two-component system", "nitrogen metabolism", "biofilm formation- $E$. coli", "lipoic acid metabolism", "sulfur relay system", "ABC transporters", "glyoxylate and dicarboxylate metabolism" (Fig. 5C), while only "ABC transporters" was significantly enriched $(P$-value $<0.05)$ in the down-regulated differential proteins (Fig. 5D).

\subsection{Cyanobacteria responses to co-culture systemdeciphered by proteomics}


As demonstrated in the transcriptomics analysis that the large fraction (25\%) of transcripts involved in photosynthesis and oxidative phosphorylation were significantly increased during co-cultivation, the differentially expressed proteins involved in the energy metabolism pathway was also identified (Suppl

Table S4). In co-cultivated Synechococcus $\operatorname{cscB}^{+}$, the increase of protein abundances for energy metabolism enzymes, such as ferredoxin (PetF, M744_01325), phycobiliproteins terminal rod linker (CpcD, M744_11425), photosystem II reaction center H (PsbH, M744_01910), photosystem II D1 protein (PsbA, M744_00850), NAD(P)H-quinone oxidoreductase subunit 4 (NadhD, M744_05920), and NAD(P)H-quinone oxidoreductase subunit 5 (NadhF, M744_01470) were observed, suggesting that more NADPH and ATP generated from photosynthesis ${ }^{57}$, which is likely due to the elevated $\mathrm{C}$ and/or $\mathrm{N}$ availability compared with the axenic control, as discussed above. Meanwhile, increased protein abundance of the lightindependent prochlorophyll reductase subunit B (ChIB) (M744_07280), which catalyzes the conversion of prochlorophyll to chlorophyll a ${ }^{58}$, was also found, suggesting that cyanobacterial photosynthesis might be improved during the co-cultivation. These results are well consistent with our findings based on the transcriptomics analysis.

Nitrogen metabolism, either from nitrate or ammonium, governs the turnovers of the macromolecules that regulate metabolic pathways, eventually affecting energy production and carbon skeleton ${ }^{59}$. Through the quantitative proteomics analysis, three nitrate/nitrite transport system ATP-binding proteins of M744_10450 (NrtB), M744_10455 (NrtC), and M744_10460 (NrtD) and two ferredoxin-nitrite reductases (M744_10440 and M744_07195) were found up-regulated in the co-cultivated Synechococcus cscB ${ }^{+}$, suggesting that the nitrite uptake in co-cultivated Synechococcus $\csc B^{+}$was enhanced. The ammonium is incorporated into carbon skeletons through glutamine synthetase (M744_02210), which was also found up-regulated in co-cultivated Synechococcus $\csc B^{+}$. Significant up-regulation in the nitrogen uptake and assimilation were evident with higher photosynthesis and better cyanobacterial growth during the cocultivation condition.

Phosphorus is a vital nutrient for cyanobacterial growth, which impacts the synthesis of cyanobacterial extracellular polymeric substances and also appears to induce significant changes in the synthesis of polysaccharides, as well as membrane lipids ${ }^{60}$. In the co-cultivated Synechococcus $\csc B^{+}$, the proteins involved in phosphate transport system, including M744_04030 (PstA), M744_04035 (PstB), M744_04025 (PstC), M744_04020 (PstS) and M744_04015 (SphX), were found up-regulated by 2.08-, 2.12-, 3.80-, 2.67- and 4.75-fold, respectively. The up-regulation of all four phosphate transporters in the co-cultivated Synechococcus $\mathrm{cscB}^{+}$might be due to the increased consumption of $\mathrm{Pi}$ in the form of NAPDH or ATP, which contributes to further cell growth. Consistently, the increased transcripts level of M744_04015 (sphX) and M744_04030 (pstA), were also found at transcription level. Aside from dissolved inorganic phosphorus, dissolved organic phosphorus is used by cyanobacteria via alkaline phosphatase 60 . Two alkaline phosphatases (M744_09635 and M744_11635) in the co-cultivated Synechococcus $\csc B^{+}$were found up-regulated by 2.89 - and 1.40 - fold, respectively, suggesting that the cyanobacteria were able to acquire more phosphorus for cell growth during the co-cultivation condition ${ }^{40}$. 
Two up-regulated proteins M744_05990 and M744_04340 annotated respectively as xylose-5phosphate/fructose 6-phosphate phosphotransketolase (Xfp) and pyruvate-ferredoxin/flavodoxin oxidoreductase (Por), were identified in the co-cultivated Synechococcus cscB ${ }^{+}$. Xfp plays a key role in glycolysis, catalyzing the conversion of X5P or F6P to acetyl phosphate ${ }^{61}$, while Por is responsible for the oxidation process of pyruvate to generate acetyl-CoA ${ }^{62}$. The up-regulation of $\mathrm{Xfp}$ and Por indicated $\mathrm{CO}_{2}$ fixation might be enhanced in the co-cultivated Synechococcus $\csc B^{+}$, well-consistent with the increased acetyl-CoA content in the metabolomic analysis discussed above. In addition, three bicarbonate transporters, including M744_08440 (CmpB), M744_08445 (CmpC), and M744_08450 (CmpD) were also found down-regulated in the quantitative proteomics data, also consistent with the transcriptomic analysis. The $c m p$ operon $(c m p A, c m p B, c m p C, c m p D)$ in Synechococcus 7942 has been confirmed to encode a component of the $\mathrm{ABC}$-type $\mathrm{HCO}^{3-}$ transporter $\mathrm{BCT} 1$, and its transcription was activated at low $\mathrm{CO}_{2}$ concentrations ${ }^{63,64}$. The down-regulation of these three bicarbonate transporters indicated that the concentration of $\mathrm{CO}_{2}$ in the co-culture system might be higher than that under pure culture conditions, due to the fact that $E$. coli $\mathrm{ABKm}$ might secret $\mathrm{CO}_{2}$ to the system, which was also found in transcriptomics.

The exchange of essential micronutrients, such as vitamins, amino acids and bioavailable trace metal, from heterotrophic bacteria to cyanobacteria during co-cultivation was observed above at the transcript level. Pathway enrichment analysis of the proteomic data indicated that Synechococcus $\operatorname{cscB}^{+}$had maximized the uptake and utilization of $\mathrm{Fe}^{3+}$ and thiamine to improve cell growth during the cocultivation condition. Significant decrease in protein abundances of $\mathrm{Fe}^{3+}$ transporter, including AfuA (M744_05470) and AfuB (M744_09555) ${ }^{65}$, indicated increased availability of this important micronutrient for $S$. elongateus $\csc B^{+}$during the co-cultivation condition. The up-regulation of thiamine metabolism proteins was also found in the co-culture system. The cysteine desulfurase (M744_03415) catalyzes the conversion of L-cysteine to L-alanine and sulfur, the released sulfur was then transferred into scaffold protein to assemble Fe-S clusters ${ }^{66}$, while the Fe-S clusters participate in electron transfer, iron-sulfur storage, regulation of gene expression, photosynthesis, and enzyme activity in all kingdoms of life ${ }^{67}$. The up-regulation of M744_03415 was observed, consistent with the cyanobacterial growth and functional performance during co-cultivation. Thiamine pyrophosphate (TPP) acts as a cofactor for several enzymes in key cellular metabolic pathways such as glycolysis, the pentose phosphate pathway and the citric acid cycle (TCA) ${ }^{68}$. Proteomic analysis showed that the phosphomethylpyrimidine synthase (ThiC) (M744_11180), an essential enzyme for TPP biosynthesis, was up-regulated in the cocultivated Synechococcus $\operatorname{cscB}^{+}$, suggesting that enhanced carbon metabolic activity in Synechococcus $\csc B^{+}$. In addition, up-regulation was also observed for 2-succinyl-5-enolpyruvyl-6-hydroxy-3-cyclohexene1-carboxylate synthase (MenD) (M744_09410), which is involved in the biosynthesis of menaquinone and phylloquinone. Menaquinones play important roles in electron transport and oxidative phosphorylation, while phyloquinone is the secondary electron carrier in the photosystem I ${ }^{69}$, their upregulation was in general consisted with the improved growth of Synechococcus $\csc B^{+}$. 
Moreover, the down-regulation of five proteins related to oxidative stress, including Hli protein (M744_01810 and M744_11065), furfamily transcription regulator (Fur) (M744_05500 and M744_12665), and monothiol glutaredoxin (M744_10930) were found in the co-cultivated Synechococcus $\operatorname{cscB}^{+}$by our quantitative proteomics analysis. The two Hli proteins were also identified in transcriptomic analysis. The transcription regulator Fur, as an iron uptake regulator, is responsible for controlling the gene expression of siderophore biosynthesis and iron transport ${ }^{70}$. In previous studies, monothiol glutaredoxin was proved to protect against oxidative stress by regulating iron homeostasis ${ }^{71}$. The crosstalk between controlling iron homeostasis and defending against ROS was previously confirmed in E. coli, demonstrating that the lack of iron regulation may lead to oxidative stress ${ }^{72}$. Thus, the down-regulation of these five proteins indicated the positive effect on cell growth during the cocultivation was attributed to the decrease of oxidative stress in Synechococcus $\csc B^{+}$by a heterotrophic cells capable of ROS scavenging, which was also consistent with the our previous finding that the gene expression of three types of catalases, including hydroperoxidase I (katG), hydroperoxidase II (katF), and hydroperoxidase III (katE) were significant induced in E.coli during the co-cultivation condition ${ }^{21}$.

\section{Conclusion}

In our previous study, we constructed a one-step conversion system from $\mathrm{CO}_{2}$, and sucrose synthesized and secreted by cyanobacteria to a fine chemical 3-HP by including a sucrose-secreting cyanobacterial Synechococcus $\operatorname{cscB}^{+}$and a 3-HP producing $E$. coli $\mathrm{ABKm}$ in a co-culture system maintaining for one week, and achieved a 3-HP production of $68.29 \mathrm{mg} / \mathrm{L}^{21}$. However, the stability and 3-HP productivity in the co-culture system are still low, probably due to the low cyanobacterial biomass and sucrose productivity in the co-culture system, and even the low metabolite exchange between the two partner cells in the system ${ }^{73}$. To further improve the functional performance of the co-culture system, an integrated omics analysis was conducted in this study to determine the interaction mechanism between cyanobacterium Synechococcus and E. coli.

The stability in the autotrophy-heterotrophy co-culture system dependents on the cyanobacterial growth and sucrose production. In this study, the decreased level of several oxidative stress related proteins was found in transcripts level (M744_11065, M744_01810, M744_03995 and M744_07160) and proteomics level (M744_01810, M744_11065, M744_05500, M744_12665 and M744_10930), suggesting the possible reduced oxidative stress in co-cultivated Synechococcus $\csc B^{+}$. In addition, the increased expression of transcript related to phospholipid/cholesterol/gamma-HCH transport system (M744_01095), and decreased expression of many transcripts involved in ribosomal proteins (M744_13675, M744_13670, M744_05205, M744_00735, M744_05210, M744_12320, M744_05195, M744_05180, M744_05185), tRNA synthetases (M744_03935, M744_5340) and RNA binding protein (M744_12800) were found in cocultivated Synechococcus $\csc B^{+}$, also suggesting that ROS induced membrane damage was relieved by the presence of the heterotrophic partner. All these finding illuminated us that the antioxidative system of E. coli could be further enhanced through overexpressing the major ROS-scavenging enzymes, for 
example superoxide dismutase, catalase, glutathione peroxidases and thioredoxin ${ }^{74}$, to improve cyanobacterial cell growth and productivity during co-cultivation. Previous studies have shown that heterotrophic species could provide essential micronutrients, such as vitamins, amino acids and bioavailable trace metals, necessary to maintain high photosynthetic productivity in various co-culture systems ${ }^{9}$. In this study, the higher availability of carbon, nitrogen, phosphate, calcium, $\mathrm{Cu}^{2+}, \mathrm{Fe}^{3+}$ and cofactors in co-cultivated Synechococcus $\operatorname{cscB}^{+}$during co-cultivation were identified by the integrated metabolomics, transcriptomics and proteomics analysis, which therefore with great promise as the potential targets to improve the fermentation performance of the co-culture system consisted with photoautotrophic and heterotrophic species.

Light condition is critical for the cell growth of cyanobacteria through photosynthesis ${ }^{75}$, especially under co-cultivation, as the cell concentration increases, light-shading caused by the heterotrophic species might reduce cyanobacterial exposure to light and thus the photosynthetic activity ${ }^{76}$. Meanwhile, the enhanced photosynthesis and oxidative phosphorylation identified by the integrated omics also illuminated light condition might be further optimized to improve the stability and efficiency of artificial co-culture system. More importantly, cyanobacteria are often inhibited by ROS produced from the imbalance between light absorption and light utilization during the process of photosynthesis ${ }^{77}$. Although in co-culture system, the inhibition of oxidative pressure on cyanobacteria can be reduced by the heterotrophic species ${ }^{13,21}$, the growth of heterotrophic species can be inhibited by ROS when exposed to high densities of cyanobacteria in the light 17,78 . Considering the light availability may influence the cross-feeding metabolites between phototrophic and heterotrophic species including oxygen and carbon dioxide through photosynthesis and respiration, and also affect the oxidative pressure on the E. coli strains due to the photosynthesis ${ }^{25}$, the optimal light conditions could also be critical to the high cell growth in the co-culture system.

In conclusion, the results showed that many metabolic changes, including enhanced photosynthesis, oxidative phosphorylation, and essential micronutrients, were discovered at multiple levels, not only the ROS scavenging, might be responsible for the better cell growth of Synechococcus $\csc B^{+}$during cocultivation (Fig. 5). We thus proposed that the metabolic modules related to the ROS quenching, carbon metabolism, nitrogen metabolism, Pi transport, metal transport and co-factors biosynthesis could be potential engineering targets to further improve stability and fermentation performance in this co-culture system.

\section{Declarations}

\section{Acknowledgements}

None.

\section{Authors' contributions}


XYS and WZ designed the research; JJM and THG performed the major experiments and wrote the draft manuscript; and JJM, THG, LC, XYS and WZ analyzed the data, drafted and revised the manuscript. All authors read and approved the final manuscript. JJM and THG contribute equally.

\section{Funding}

This research was supported by Grants from the National Key Research and Development Program of China (Grants No. 2019YFA0904600, 2018YFA0903000, 2018YFA0903600 and 2020YFA0906800), the National Natural Science Foundation of China (nos. 31901016, 31770035, 31770100, 31972931, 91751102 and 21621004), and Tianjin Synthetic Biotechnology Innovation Capacity Improvement Project (no. TSBICIP-KJGG-007).

\section{Availability of supporting data}

All data generated or analyzed during this study are included in this published article and its additional files.

\section{Ethics approval and consent to participate}

Not applicable.

\section{Consent for publication}

All authors have agreed to the publication of this manuscript.

\section{Competing interests}

The authors declare no competing financial interests.

\section{References}

1. Quintana, N.; Van der Kooy, F.; Van de Rhee, M. D.; Voshol, G. P.; Verpoorte, R., Renewable energy from cyanobacteria: energy production optimization by metabolic pathway engineering. Appl Microbiol Biotechnol 2011, 91 (3), 471-90.

2. Hays, S. G.; Ducat, D. C., Engineering cyanobacteria as photosynthetic feedstock factories. Photosynth Res 2015, 123 (3), 285-295.

3. Hagemann, M., Molecular biology of cyanobacterial salt acclimation. FEMS Microbiol Rev 2011, 35 (1), 87-123.

4. Niederholtmeyer, H.; Wolfstadter, B. T.; Savage, D. F.; Silver, P. A.; Way, J. C., Engineering cyanobacteria to synthesize and export hydrophilic products. Appl Environ Microbiol 2010, 76 (11), 3462-6.

5. Han, Y.; Watson, M., Production of microbial levan from sucrose, sugarcane juice and beet molasses. $\mathrm{J}$ Ind Microbiology 1992, 9 (3), 257-260.

6. Chisti, Y., Constraints to commercialization of algal fuels. J Biotechnol 2013, 167 (3), 201-14. 
7. Luan, G.; Lu, X., Tailoring cyanobacterial cell factory for improved industrial properties. Biotechnol Adv 2018, 36 (2), 430-442.

8. Beliaev, A. S.; Romine, M. F.; Serres, M.; Bernstein, H. C.; Linggi, B. E.; Markillie, L. M.; Isern, N. G.; Chrisler, W. B.; Kucek, L. A.; Hill, E. A., Inference of interactions in cyanobacterial-heterotrophic cocultures via transcriptome sequencing. ISME J 2014, 8 (11), 2243-2255.

9. Giri, S.; Ona, L.; Waschina, S.; Shitut, S.; Yousif, G.; Kaleta, C.; Kost, C., Metabolic dissimilarity determines the establishment of cross-feeding interactions in bacteria. Curr Biol 2021.

10. Paerl, H. W.; Pinckney, J., A mini-review of microbial consortia: their roles in aquatic production and biogeochemical cycling. Microbial Ecology 1996, 31 (3), 225-247.

11. Bruckner, C. G.; Rehm, C.; Grossart, H. P.; Kroth, P. G., Growth and release of extracellular organic compounds by benthic diatoms depend on interactions with bacteria. Environ Microbiol 2011, 13 (4), 1052-63.

12. Seymour, J. R.; Ahmed, T.; Durham, W. M.; Stocker, R., Chemotactic response of marine bacteria to the extracellular products of Synechococcus and Prochlorococcus. Aquat Microb Ecol 2010, 59 (2), 161168.

13. Li, T.; Li, C. T.; Butler, K.; Hays, S. G.; Guarnieri, M. T.; Oyler, G. A.; Betenbaugh, M. J., Mimicking lichens: incorporation of yeast strains together with sucrose-secreting cyanobacteria improves survival, growth, ROS removal, and lipid production in a stable mutualistic co-culture production platform. Biotechnol Biofuels 2017, 10, 55.

14. Morris, J. J.; Kirkegaard, R.; Szul, M. J.; Johnson, Z. I.; Zinser, E. R., Facilitation of robust growth of Prochlorococcus colonies and dilute liquid cultures by "helper" heterotrophic bacteria. Appl Environ Microbiol 2008, 74 (14), 4530-4.

15. Brenner, K.; You, L.; Arnold, F. H., Engineering microbial consortia: a new frontier in synthetic biology. Trends Biotechnol 2008, 26 (9), 483-9.

16. Fredrickson, J. K., ECOLOGY. Ecological communities by design. Science 2015, 348 (6242), 1425-7.

17. Hays, S. G.; Yan, L. L. W.; Silver, P. A.; Ducat, D. C., Synthetic photosynthetic consortia define interactions leading to robustness and photoproduction. J Biol Eng 2017, 11, 4.

18. Weiss, T. L.; Young, E. J.; Ducat, D. C., A synthetic, light-driven consortium of cyanobacteria and heterotrophic bacteria enables stable polyhydroxybutyrate production. Metab Eng 2017, 44, 236-245.

19. Liu, H.; Cao, Y.; Guo, J.; Xu, X.; Long, Q.; Song, L.; Xian, M., Study on the isoprene-producing co-culture system of Synechococcus elongates-Escherichia coli through omics analysis. Microb Cell Fact 2021, $20(1), 6$.

20. Yu, J. J.; Liberton, M.; Cliften, P. F.; Head, R. D.; Jacobs, J. M.; Smith, R. D.; Koppenaal, D. W.; Brand, J. J.; Pakrasi, H. B., Synechococcus elongatus UTEX 2973, a fast growing cyanobacterial chassis for biosynthesis using light and $\mathrm{CO}_{2}$. Sci Rep-Uk 2015, 5 .

21. Zhang, L.; Chen, L.; Diao, J.; Song, X.; Shi, M.; Zhang, W., Construction and analysis of an artificial consortium based on the fast-growing cyanobacterium Synechococcus elongatus UTEX 2973 to 
produce the platform chemical 3-hydroxypropionic acid from $\mathrm{CO}_{2}$. Biotechnol Biofuels 2020, 13, 82.

22. Bernstein, H. C.; McClure, R. S.; Thiel, V.; Sadler, N. C.; Kim, Y. M.; Chrisler, W. B.; Hill, E. A.; Bryant, D. A.; Romine, M. F.; Jansson, J. K.; Fredrickson, J. K.; Beliaev, A. S., Indirect interspecies regulation: Transcriptional and physiological responses of a cyanobacterium to heterotrophic partnership. mSystems 2017, 2 (2).

23. Bobadilla Fazzini, R. A.; Preto, M. J.; Quintas, A. C.; Bielecka, A.; Dos Santos, V. A., Consortia modulation of the stress response: proteomic analysis of single strain versus mixed culture. Environ Microbiol 2010, 12 (9), 2436-49.

24. Nouaille, S.; Even, S.; Charlier, C.; Le Loir, Y.; Cocaign-Bousquet, M.; Loubiere, P., Transcriptomic response of Lactococcus lactis in mixed culture with Staphylococcus aureus. Appl Environ Microbiol 2009, 75 (13), 4473-82.

25. Liu, H.; Cao, Y.; Guo, J.; Xu, X.; Long, Q.; Song, L.; Xian, M., Study on the isoprene-producing co-culture system of Synechococcus elongates-Escherichia coli through omics analysis. Microb Cell Fact 2021, 20 (1), 1-18.

26. Amin, S.; Hmelo, L.; Van Tol, H.; Durham, B.; Carlson, L.; Heal, K.; Morales, R.; Berthiaume, C.; Parker, M.; Djunaedi, B., Interaction and signalling between a cosmopolitan phytoplankton and associated bacteria. Nature 2015, 522 (7554), 98-101.

27. Stanier, R. Y.; Kunisawa, R.; Mandel, M.; Cohen-Bazire, G., Purification and properties of unicellular blue-green algae (order Chroococcales). Bacteriol Rev 1971, 35 (2), 171-205.

28. Jeong, S. Y.; Kim, T. G., Development of a novel methanotrophic process with the helper microorganism Hyphomicrobium sp. NM3. J Appl Microbiol 2019, 126 (2), 534-544.

29. Song, X.; Diao, J.; Yao, J.; Cui, J.; Sun, T.; Chen, L.; Zhang, W., Engineering a central carbon metabolism pathway to increase the intracellular acetyl-CoA pool in Synechocystis sp. PCC 6803 grown under photomixotrophic conditions. ACS Synth Biol 2021, 10 (4), 836-846.

30. Gao, W.; Zhang, W.; Meldrum, D. R., RT-qPCR based quantitative analysis of gene expression in single bacterial cells. J Microbiol Methods 2011, 85 (3), 221-7.

31. Livak, K. J.; Schmittgen, T. D., Analysis of relative gene expression data using real-time quantitative PCR and the 2(-Delta Delta C(T)) Method. Methods 2001, 25 (4), 402-8.

32. Fischer, B. B.; Hideg, E.; Krieger-Liszkay, A., Production, detection, and signaling of singlet oxygen in photosynthetic organisms. Antioxid Redox Signal 2013, 18 (16), 2145-62.

33. Chen, L.; Xie, M.; Bi, Y.; Wang, G.; Deng, S.; Liu, Y., The combined effects of UV-B radiation and herbicides on photosynthesis, antioxidant enzymes and DNA damage in two bloom-forming cyanobacteria. Ecotoxicol Environ Saf 2012, 80, 224-30.

34. Singh, S. P.; Montgomery, B. L., Reactive oxygen species are involved in the morphology-determining mechanism of Fremyella diplosiphon cells during complementary chromatic adaptation. Microbiol (Reading) 2012, 158 (Pt 9), 2235-2245. 
35. Gao, L.; Pan, X.; Zhang, D.; Mu, S.; Lee, D. J.; Halik, U., Extracellular polymeric substances buffer against the biocidal effect of $\mathrm{H}_{2} \mathrm{O}_{2}$ on the bloom-forming cyanobacterium Microcystis aeruginosa. Water Res 2015, 69, 51-58.

36. Wang, Y.; Chen, L.; Zhang, W., Proteomic and metabolomic analyses reveal metabolic responses to 3hydroxypropionic acid synthesized internally in cyanobacterium Synechocystis sp. PCC 6803. Biotechnol Biofuels 2016, 9, 209.

37. Shu, C.-H.; Tsai, C.-C.; Chen, K.-Y.; Liao, W.-H.; Huang, H.-C., Enhancing high quality oil accumulation and carbon dioxide fixation by a mixed culture of Chlorella sp. and Saccharomyces cerevisiae. J Taiwan Inst Chem Eng 2013, 44 (6), 936-942.

38. Lin, P. C.; Zhang, F.; Pakrasi, H. B., Enhanced production of sucrose in the fast-growing cyanobacterium Synechococcus elongatus UTEX 2973. Sci Rep 2020, 10 (1), 390.

39. Beliaev, A. S.; Romine, M. F.; Serres, M.; Bernstein, H. C.; Linggi, B. E.; Markillie, L. M.; Isern, N. G.; Chrisler, W. B.; Kucek, L. A.; Hill, E. A.; Pinchuk, G. E.; Bryant, D. A.; Wiley, H. S.; Fredrickson, J. K.; Konopka, A., Inference of interactions in cyanobacterial-heterotrophic co-cultures via transcriptome sequencing. ISME J 2014, 8 (11), 2243-2255.

40. Merchant, S. S.; Helmann, J. D., Elemental economy: Microbial strategies for optimizing growth in the face of nutrient limitation. Adv Microb Physiol 2012, 60, 91-210.

41. Zahalak, M.; Pratte, B.; Werth, K. J.; Thiel, T., Molybdate transport and its effect on nitrogen utilization in the cyanobacterium Anabaena variabilis ATCC 29413. Mol Microbiol 2004, 51 (2), 539-49.

42. Varkey, D.; Mazard, S.; Ostrowski, M.; Tetu, S. G.; Haynes, P.; Paulsen, I. T., Effects of low temperature on tropical and temperate isolates of marine Synechococcus. ISME J 2016, 10 (5), 1252-63.

43. Latifi, A.; Ruiz, M.; Zhang, C.-C., Oxidative stress in cyanobacteria. FEMS Microbiol Rev 2009, 33 (2), 258-278.

44. He, Q. F.; Dolganov, N.; Bjorkman, 0.; Grossman, A. R., The high light-inducible polypeptides in Synechocystis PCC6803 - Expression and function in high light. J Bio Chem 2001, 276 (1), 306-314.

45. Cornejo, J.; Willows, R. D.; Beale, S. I., Phytobilin biosynthesis: cloning and expression of a gene encoding soluble ferredoxin-dependent heme oxygenase from Synechocystis sp. PCC 6803. Plant J 1998, 15 (1), 99-107.

46. Cheng, D.; He, Q., PfsR is a key regulator of iron homeostasis in Synechocystis PCC 6803. PLoS One 2014, 9 (7), e101743.

47. Vance, J. E., Phospholipid synthesis and transport in mammalian cells. Traffic 2015, 16 (1), 1-18.

48. Frain, K. M.; Gangl, D.; Jones, A.; Zedler, J. A.; Robinson, C., Protein translocation and thylakoid biogenesis in cyanobacteria. Biochim Biophys Acta 2016, 1857 (3), 266-73.

49. de Keyzer, J.; van der Does, C.; Driessen, A. J., The bacterial translocase: a dynamic protein channel complex. Cell Mol Life Sci 2003, 60 (10), 2034-52.

50. Fulda, S.; Huang, F.; Nilsson, F.; Hagemann, M.; Norling, B., Proteomics of Synechocystis sp strain PCC 6803 - Identification of periplasmic proteins in cells grown at low and high salt concentrations. Eur $\mathrm{J}$ 
Biochem 2000, 267 (19), 5900-5907.

51. Wang, P.; Dalbey, R. E., Inserting membrane proteins: The YidC/Oxa1/Alb3 machinery in bacteria, mitochondria, and chloroplasts. Bba-Biomembranes 2011, 1808 (3), 866-875.

52. Edhofer, I.; Muhlbauer, S. K.; Eichacker, L. A., Light regulates the rate of translation elongation of chloroplast reaction center protein D1. Eur J Biochem 1998, 257 (1), 78-84.

53. Nie, L.; Wu, G.; Culley, D. E.; Scholten, J. C.; Zhang, W., Integrative analysis of transcriptomic and proteomic data: challenges, solutions and applications. Crit Rev Biotechnol 2007, 27 (2), 63-75.

54. Zhang, W. W.; Li, F.; Nie, L., Integrating multiple 'omics' analysis for microbial biology: application and methodologies. Microbiol-Sgm 2010, 156, 287-301.

55. Nie, L.; Wu, G.; Zhang, W., Correlation of mRNA expression and protein abundance affected by multiple sequence features related to translational efficiency in Desulfovibrio vulgaris: a quantitative analysis. Genetics 2006, 174 (4), 2229-43.

56. Ruffing, A. M., RNA-Seq analysis and targeted mutagenesis for improved free fatty acid production in an engineered cyanobacterium. Biotechnol Biofuels 2013, 6.

57. Battchikova, N.; Aro, E. M., Cyanobacterial NDH-1 complexes: multiplicity in function and subunit composition. Physiol Plantarum 2007, 131 (1), 22-32.

58. Corless, E. I.; Bennett, B.; Antony, E., Substrate recognition induces sequential electron transfer across subunits in the nitrogenase-like DPOR complex. J Biol Chem 2020, 295 (39), 13630-13639.

59. Smith, S. R.; Dupont, C. L.; McCarthy, J. K.; Broddrick, J. T.; Obornik, M.; Horak, A.; Fussy, Z.; Cihlar, J.; Kleessen, S.; Zheng, H.; McCrow, J. P.; Hixson, K. K.; Araujo, W. L.; Nunes-Nesi, A.; Fernie, A.; Nikoloski, Z.; Palsson, B. O.; Allen, A. E., Evolution and regulation of nitrogen flux through compartmentalized metabolic networks in a marine diatom. Nat Commun 2019, 10.

60. Lin, W.; Zhao, D.; Luo, J., Distribution of alkaline phosphatase genes in cyanobacteria and the role of alkaline phosphatase on the acquisition of phosphorus from dissolved organic phosphorus for cyanobacterial growth. J Appl Phycol 2018, 30 (2), 839-850.

61. Glenn, K.; Smith, K. S., Allosteric regulation of Lactobacillus plantarum xylulose 5phosphate/fructose 6-phosphate phosphoketolase (Xfp). J Bacteriol 2015, 197 (7), 1157-63.

62. Kletzin, A.; Adams, M. W., Molecular and phylogenetic characterization of pyruvate and 2ketoisovalerate ferredoxin oxidoreductases from Pyrococcus furiosus and pyruvate ferredoxin oxidoreductase from Thermotoga maritima. J Bacteriol 1996, 178 (1), 248-57.

63. Omata, T.; Takahashi, Y.; Yamaguchi, O.; Nishimura, T., Structure, function and regulation of the cyanobacterial high-affinity bicarbonate transporter, BCT1. Funct Plant Biol 2002, 29 (3), 151-159.

64. Nishimura, T.; Takahashi, Y.; Yamaguchi, O.; Suzuki, H.; Maeda, S.-i.; Omata, T., Mechanism of low $\mathrm{CO}_{2}$-induced activation of the $\mathrm{cmp}$ bicarbonate transporter operon by a LysR family protein in the cyanobacterium Synechococcus elongatus strain PCC 7942. Mol Microbiol 2008, 68 (1), 98-109.

65. Adhikari, P.; Berish, S. A.; Nowalk, A. J.; Veraldi, K. L.; Morse, S. A.; Mietzner, T. A., The fbpABC locus of Neisseria gonorrhoeae functions in the periplasm-to-cytosol transport of iron. J Bacteriol 1996, 178 
(7), 2145-9.

66. Mihara, H.; Esaki, N., Bacterial cysteine desulfurases: their function and mechanisms. Appl Microbiol Biotechnol 2002, 60 (1-2), 12-23.

67. Johnson, D. C.; Dean, D. R.; Smith, A. D.; Johnson, M. K., Structure, function, and formation of biological iron-sulfur clusters. Annu Rev Biochem 2005, 74, 247-81.

68. Pohl, M.; Sprenger, G. A.; Muller, M., A new perspective on thiamine catalysis. Curr Opin Biotechnol 2004, 15 (4), 335-42.

69. Furt, F.; Oostende, C.; Widhalm, J. R.; Dale, M. A.; Wertz, J.; Basset, G. J., A bimodular oxidoreductase mediates the specific reduction of phylloquinone (vitamin K区) in chloroplasts. Plant J 2010, 64 (1), 38-46.

70. Kaushik, M. S.; Singh, P.; Tiwari, B.; Mishra, A. K., Ferric uptake regulator (FUR) protein: properties and implications in cyanobacteria. Ann Microbiol 2016, 66 (1), 61-75.

71. Pujol-Carrion, N.; de la Torre-Ruiz, M. A., Glutaredoxins Grx4 and Grx3 of Saccharomyces cerevisiae play a role in actin dynamics through their trx domains, which contributes to oxidative stress resistance. Appl Environ Microbiol 2010, 76 (23), 7826-7835.

72. Lee, J. W.; Helmann, J. D., Functional specialization within the Fur family of metalloregulators. Biometals 2007, 20 (3-4), 485-99.

73. Du, Y.; Zou, W.; Zhang, K.; Ye, G.; Yang, J., Advances and applications of clostridium co-culture systems in biotechnology. Front Microbiol 2020, 11, 560223.

74. He, L.; He, T.; Farrar, S.; Ji, L.; Liu, T.; Ma, X., Antioxidants maintain cellular redox homeostasis by elimination of reactive oxygen species. Cell Physiol Biochem 2017, 44 (2), 532-553.

75. Xue, S.; Su, Z.; Cong, W., Growth of Spirulina platensis enhanced under intermittent illumination. J Biotechnol 2011, 151 (3), 271-7.

76. Bohmer, S.; Koninger, K.; Gomez-Baraibar, A.; Bojarra, S.; Mugge, C.; Schmidt, S.; Nowaczyk, M. M.; Kourist, R., Enzymatic oxyfunctionalization driven by photosynthetic water-splitting in the cyanobacterium Synechocystis sp PCC 6803. Catalysts 2017, 7 (8).

77. Rinalducci, S.; Pedersen, J. Z.; Zolla, L., Generation of reactive oxygen species upon strong visible light irradiation of isolated phycobilisomes from Synechocystis PCC 6803. Bba-Bioenergetics 2008, 1777 (5), 417-424.

78. Imlay, J. A., The molecular mechanisms and physiological consequences of oxidative stress: lessons from a model bacterium. Nat Rev Microbiol 2013, 11 (7), 443-54.

\section{Figures}



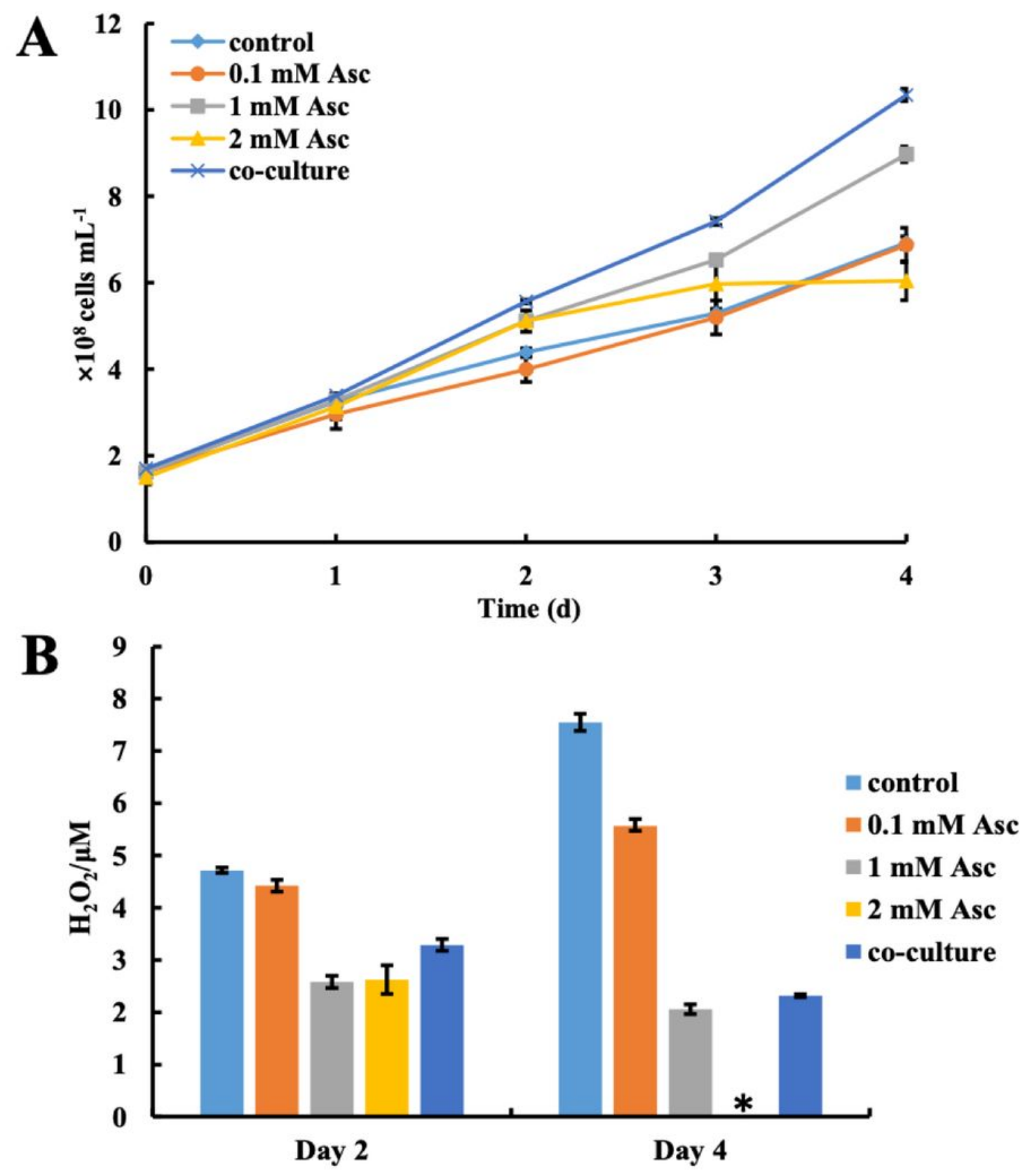

\section{Figure 1}

Analysis of the effect of quenching ROS on cultivated cyanobacterial cell growth by adding ascorbic acid. Cell growth curves of Synechococcus $\operatorname{cscB}+(A)$ and $\mathrm{H} 2 \mathrm{O} 2$ content $(B)$ in co-culture system and axenic culture with additional ascorbic acid.

\section{Figure 2}

Target metabolomics analysis of Synechococcus cscB+ under co-culture and axenic culture condition. (A) The metabolites change in central metabolic pathway in co-cultivated Synechococcus cscB+ 
compared with axenic culture condition; (B) Heatmaps of metabolomics profiles in Synechococcus cscB+ under co-culture and axenic culture condition. Each color on the heatmap corresponds to a concentration value. The higher the concentration, the darker the color (red represents the increase, and green represents the decrease).

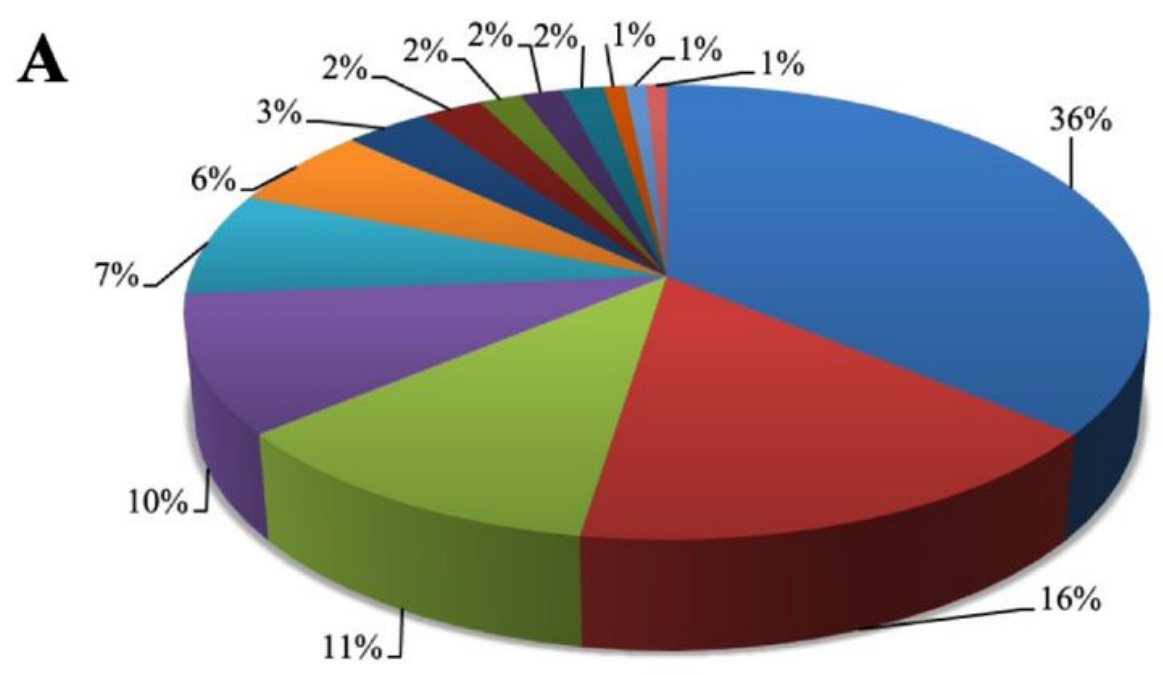

\author{
- Function unknown \\ - Photosynthesis \\ ๓ Transpoter \\ - Translation \\ = Oxidative phosphorylation \\ m Genetic information processing \\ - Metabolism of cofactors and vitamins \\ - Signaling transduction \\ - Lipid metabolism \\ - Carbohydrate metabolism \\ - Carbohydrate metabolism \\ - Nitrogen metabolism \\ - Amino acid metabolism \\ - Glycan biosynthesis and metabolism
}

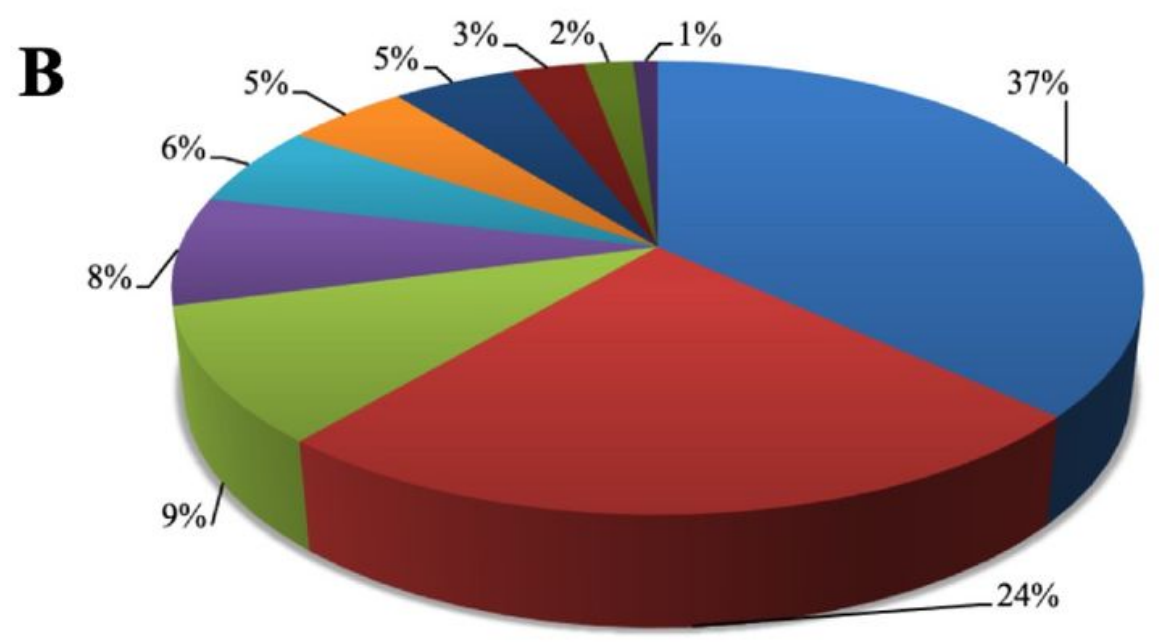

- Function unknown

- Genetic information processing

= Signaling transduction

- Amino acid metabolism

= Bacterial motility proteins

$\approx$ Photosynthesis

- Transporter

- Metabolism of cofactors and vitamins

n Oxidative phosphorylation

- Carbohydrate metabolism

\title{
Figure 3
}

Pathway classification distribution of differentially expressed genes in co-cultivated Synechococcus cscB+ compared grew under pure culture condition. (A) Up-regulated genes KEGG pathway analysis; (B) Down-regulated genes KEGG pathway analysis. KEGG, Kyoto Encyclopedia of Genes and Genomes. 

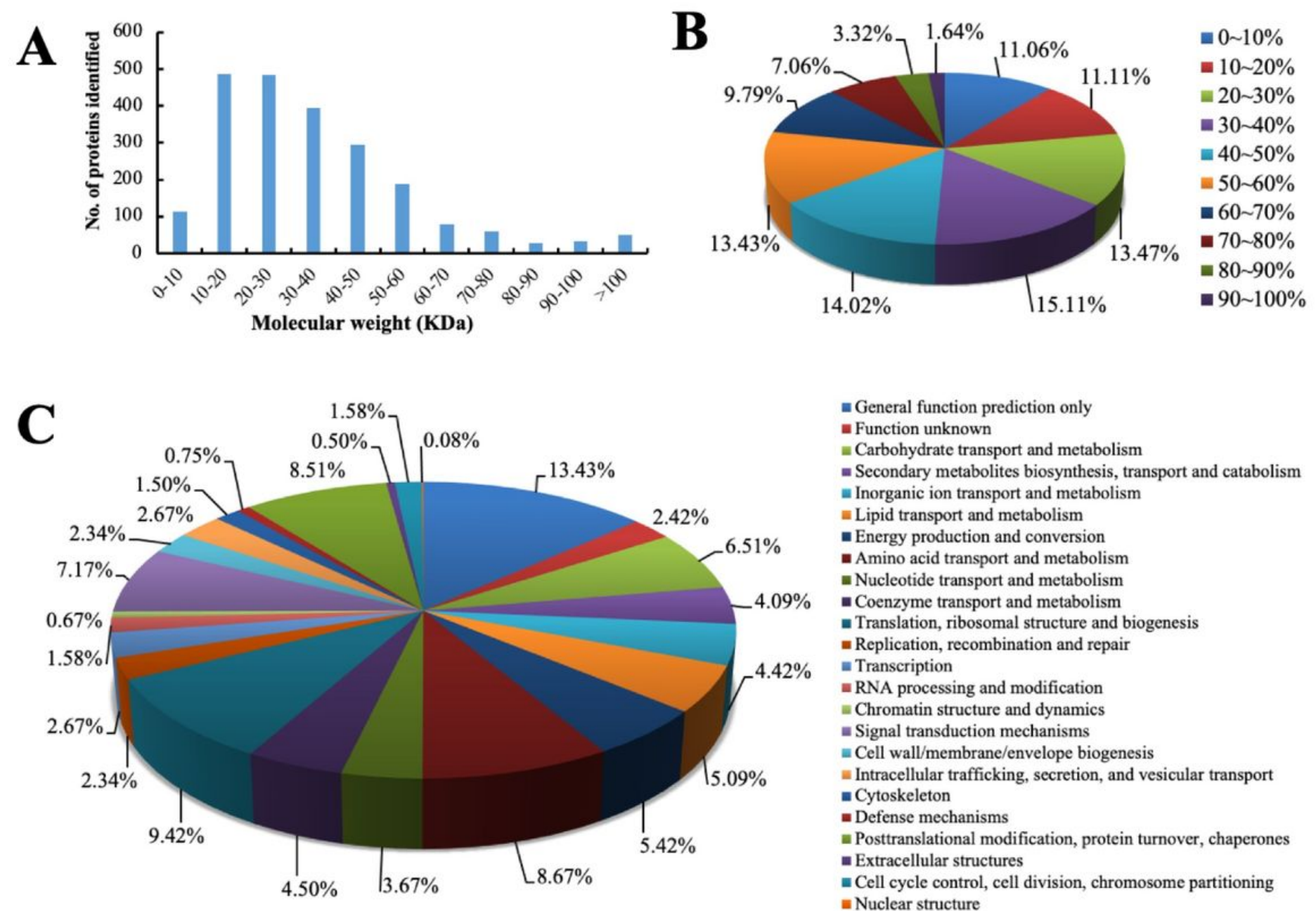

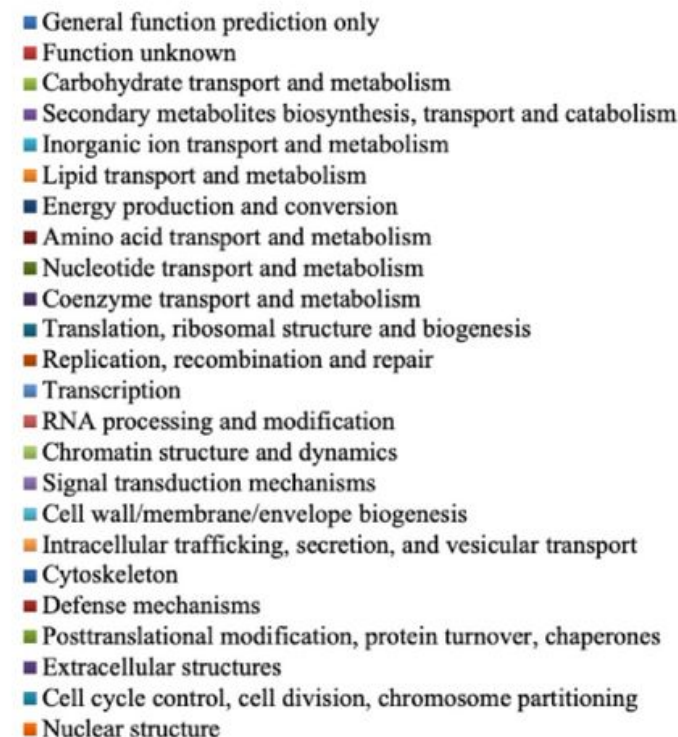

\section{Figure 4}

Distribution, coverage, and functional category of proteins identified in this study. (A) Distribution of protein identified among different molecular weights; (B) Coverage of proteins by the identified peptides; (C) Functional category coverage of the proteins identified. 


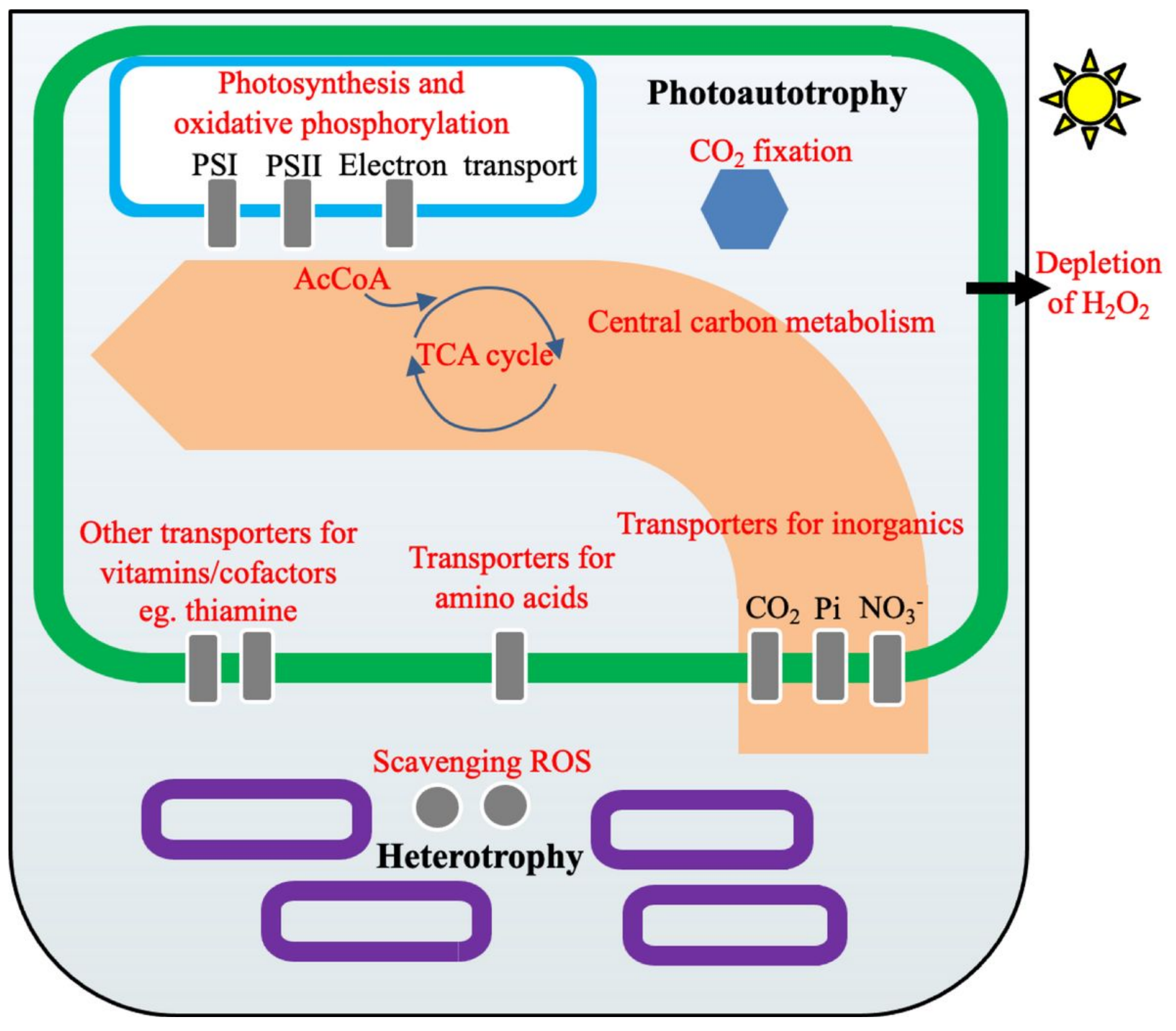

Figure 5

Schematic representation of the cross-feeding process from heterotrophic to photoautotrophic species occurred in the artificial co-culture system.

\section{Supplementary Files}

This is a list of supplementary files associated with this preprint. Click to download.

- Fig.S1.jpg

- Fig.S2.jpg 